\title{
US-amerikanische Empfehlungen zur Senkung der Kaiserschnittrate
}

\author{
In der vorherigen Ausgabe von Die Hebamme (2/2014) hat Christiane Schwarz die aktu- \\ ellen US-amerikanischen Empfehlungen vorgestellt unter dem Titel „Dem Wahnsinn \\ Einhalt gebieten?" und mit der Prognose, dass sie die geburtshilfliche Welt verändern. \\ Wir haben Hebammen und Frauenärzte gefragt, wie sie die Bedeutung dieser Leitlinie \\ für Deutschland einschätzen. Wir freuen uns auf eine rege Fachdiskussion.
}

\section{Es geschehen noch Zeichen und Wunder}

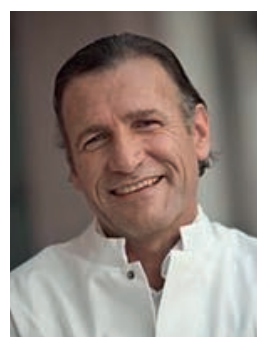

\author{
Dr. med. W. Lütje \\ Ev. Amalie Sieveking-Krankenhaus, \\ Klinik für Gynäkologie und Geburtshilfe \\ Haselkamp 33 \\ 22359 Hamburg \\ E-Mail: w.luetje@amalie.de
}

Ausgerechnet die Amerikaner machen sich intensive und höchst revolutionäre Gedanken zur Senkung der Kaiserschnittrate. Christiane Schwarz stellt in ihrem Artikel die neue Leitlinie des American College of Obstetricians \& Gynecologist (ACOG) vor, die man - mit Abstrichen und Ergänzungen - durchaus auch in Deutschland veröffentlichen könnte.

Interessanterweise entwickelt sich auch in Deutschland eine ähnliche Diskussion - erst unlängst auf einer Tagung des Arbeitskreises Frauengesundheit in Medizin, Psychotherapie und Gesellschaft e.V. (AKF) im Juni 2014 in Berlin. Anwesend waren wichtige Meinungsbildner aus allen Bereichen des Gesundheitswesens. Der Tenor deckte sich mit dem der amerikanischen Leitlinie.

Die Hauptgründe für die steigende Sectio-Rate in Deutschland, die auch bei uns nachweislich regional sehr unterschiedlich ist, sind: Die Ausweitung der ,weichen Indikationen', unzureichende Finanzierung, zunehmender forensischer Druck, unzureichende Betreuung von Gebärenden und nicht zuletzt ein gesellschaftlicher Paradigmenwechsel. Hinzu kommt ein dramatischer Wissensverlust bei den Geburtshelfern. Zudem sollte man die Effekte durch die vereinfachte Operationstechnik und gut gemeinte, aber kontraproduktive Surrogate wie die ,Kaisergeburt' nicht unterschätzen.

Auf der Metaebene könnte man diesen Trends drei Maßnahmen entgegenhalten:

\section{Sinnstiftung}

Derzeit gibt es keine wissenschaftliche Theorie über die normale physiologische Geburt. Obwohl es fast absurd erscheint, ist dies vor dem Hintergrund einer sich alternativ entwickelnden Geburtsform wie dem Kaiserschnitt dringend erforderlich. Der Sinn des Gebärens, so plausibel er Menschen mit gesundem Verstand erscheint, muss neu gefunden bzw. erfunden werden. Dies geht nur über die Wissenschaft. Viele erinnern sich an eine ähnliche Entwicklung beim Thema Stillen. Schon totgesagt hat es durch eine hohe Wissenschaftlichkeit eine Renaissance erlebt. Ähnliches muss für die Geburt nachvollzogen werden.

\section{Entängstigung}

In der Forensik spielt in der Regel nur der zu spät bzw. gar nicht durchgeführte Kaiserschnitt eine Rolle. Dies muss sich ändern. Viele Geburtshelfer ziehen sich, zum Teil auch persönlich betroffen, auf das operative Terrain zurück. Dabei geht viel geburtshilfliches Wissen verloren. Angst ist kein guter Geburtshelfer. Es sollte in der Geburtshilfe immer nur um Demut, Respekt und Verantwortung gehen. Rechtlich wäre es möglich, die teilweise entwürdigenden Haftpflichtverfahren zu entschärfen, indem ein sogenannter Regulierungsfond eingerichtet wird. Hierzu brauchen wir eine politische Entscheidung. Ich bin mir sicher, dass sich in diesem Lande - entängstigt und unterstützt von einer neuen Leitlinie - wieder mutige Geburtshelfer finden werden, die ihr Handwerk weiter verstehen. 


\section{Betreuung}

Die Eins-zu-eins-Betreuung ist bekanntlich der beste geburtsprotektive Faktor. Sie kostet nachweislich Geld! Kostenträger und politisch Verantwortliche betonen zu Recht, dass neue Formen der Finanzierung erst dann diskutiert werden können, wenn in einer neuen Leitlinie die Bedeutung der Betreuung hervorgehoben und ihr Einfluss auf die Sectiorate benannt wird.

\section{Was braucht es also in diesem Lande?}

Es wird höchste Zeit für eine neue umfassende Leitlinie zur Sectio. Prof. Frank Louwen aus Frankfurt wurde als neuer Leitlinienkoordinator benannt. Es besteht große Hoffnung, dass unter seiner Federführung die deutsche Leitlinie ein amerikanisches Gewand bekommt. Gerade die Anzahl primärer, insbesondere geplanter Kaiserschnitte muss verringert werden. Dies ist ein erster wichtiger Schritt.

Problematisch ist, dass die Entwicklung einer S3-Leitlinie ca. $200.000 €$ kostet. Da scheint es sehr sinnvoll, eine entsprechende Stiftung einzurichten! Solange eine solche Leitlinie mit einem völlig neuen Tenor nicht veröffentlicht ist, wird sich in Deutschland nicht viel ändern. Daher sind alle, die an der natürlichen Geburt festhalten möchten, aufgerufen, dieses LeitlinienProjekt ideell und wenn möglich auch finanziell zu unterstützen.

\section{Kritik}

Kritisch möchte ich folgendes zur aktuellen US-amerikanischen Leitlinie anmerken:

So erfreulich die Verlängerung der Geburtsphasen ist, vor allem die Verschiebung der Grenze zwischen Latenz- und Aktivphase: In einer Zeit, in der Kontrolle und nicht Hingabe das Geburtsgeschehen begleiten, wird es den vielen erschöpften, geburtsmüden Frauen nicht vermittelbar sein, noch längere Geburtszeiten in Kauf zu nehmen. Deshalb sollten schon in der Schule neue Weichen gestellt werden, um Frauen und auch Männer auf die Physiologie der Geburt einzustimmen. Nebenbei sei an dieser Stelle erwähnt, dass ich die gezielte Schwangerschafts- und Geburtsvorbereitung von Männern für einen wichtigen Schlüssel im Einsatz für die normale Geburt halte. Gut vorbereitete und eingestimmte Männer sind ein weiterer wichtiger geburtsproduktiver Faktor.

Die Empfehlung, eine Geburt ab der 41. + 0 SSW einzuleiten, sehe ich kritisch. Wer weiß, wie psychologisch belastend eine Einleitung ist, kann ca. 25\% mehr Einleitungen nicht ohne weiteres zustimmen. Frustrane Einleitungen enden oft mit einer primären Sectio, die es doch eigentlich zu vermeiden gilt. Ich bin gespannt, wie wir uns in Deutschland positionieren werden.
Leider fehlen in der amerikanischen Leitlinie Stellungnahmen zur Problematik der primären Sectio und zum Geburtsmanagement bei Z.n. Sectio. Diese Themen werden uns bei der Neufassung der deutschen Leitlinie bestimmt sehr beschäftigen.

\section{Fazit}

Wenn die Amerikaner medizinischen Handlungsbedarf sehen, setzen sie diesen relativ schnell und radikal um. Davon können wir lernen. Selbstredend muss eine neue Leitlinie wohlüberlegt sein. Leider wird es in Deutschland viel Widerstand gegen Veränderungen geben, weil massiv Interessen berührt werden. Trotzdem weht auch in Deutschland ein neuer Wind, der politische Unterstützung finden könnte. Eine neue, ausreichend finanzierte Leitlinie könnte der Schlüssel werden, die Sectiorate in Deutschland wieder weit unter die 30-Prozent-Marke zu drücken. Wenn die Sectiorate regional zum Qualitätsindikator wird (und werden muss!), werden wieder eindeutige medizinische Kriterien die Indikationen für den Kaiserschnitt bestimmen. Der Kaiserschnitt kann dann wieder zu dem werden, was er eigentlich ist: eine großartige Methode, um dafür zu sorgen, dass Familien an Leib und Seele gesund bleiben. 


\section{Persönliche Gedanken}

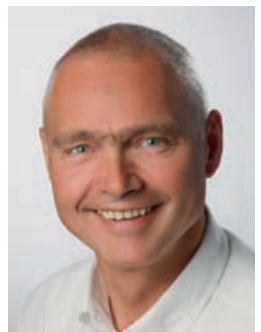

\author{
Dr. med. Michael Krause \\ Klinikum Nürnberg, Klinik für Frauen- \\ heilkunde/Bereich Geburtshilfe \\ Breslauer Str. 201 \\ 90471 Nürnberg \\ E-Mail: \\ michael.krause@klinikum-nuernberg.de
}

Die Entwicklung der Sectiorate der letzten 25 Jahre ist sehr beklagenswert. Bei meinem Eintritt in das Nürnberger Perinatalzentrum im Jahr 1991 betrug die Sectiorate weniger als 10\%, 22 Jahre später 24,2\%, in Bayern 33,2\% (BAQ-Jahresauswertung 2013)! Obwohl sich die Sectiorate auch in unserer Klinik mehr als verdoppelt hat, haben sich die Raten schwerer fetaler Azidosen, die Verlegungsraten von Neugeborenen auf die neonatologische Intensivstation, das Vorkommen kindlicher Todesfälle oder schwerer neonatologischer Erkrankungen gemäß unserer Klinikstatistik seit vielen Jahren nicht wesentlich verändert. Somit schlussfolgere ich, dass die „weich“ indizierte Sectio, ob primär oder sekundär, keinen oder nur einen marginalen Effekt auf die Verbesserung des kindlichen Outcomes hat.

Die Sectio caesarea, medizinisch korrekt indiziert, ist ein Segen für Mutter und Kind - ganz ohne Zweifel! Aber jenseits der 15\%-Rate bringt sie keinen Gesundheitsvorteil für Mutter und Kind. Daher begrüße ich es sehr, dass sich die amerikanischen Geburtsmediziner Gedanken über die vermeidbar hohen primären Sectioraten machen! Es ist längst überfällig, diese Fehlentwicklung zu stoppen und zu den medizinisch-wissenschaftlich begründeten Indikationen für die Sectio zurückzukehren. Ich kann mir vorstellen, dass sich die deutschen Geburtsmediziner der US-amerikanischen Sichtweise anschließen und sich gegen die allgemein akzeptierten „weichen“ Indikationen zur primären Sectio noch stärker positionieren. Konsequenter Weise müsste dann die deutsche Leitlinie „Absolute und relative Indikationen zur Sectio caesarea und zur Frage der sogenannten Sectio auf Wunsch“ aktualisiert werden. Obwohl Unterschiede in den jeweiligen Gesundheitssystemen bestehen, sind die medizinischen Gründe für die primären Sectiones in unseren Ländern nicht verschieden.

\section{Mangel an Eins-zu-Eins-Betreuung}

Begrenzte finanzielle Ressourcen und starker finanzieller Druck führen zu einem Überlebenskampf der Krankenhäuser, in dem alle Mittel recht sind. Auswirkungen hat diese Entwicklung z.B. auf die Stellenpläne der Frauenkliniken. Die notwendige und begründete Eins-zu-Eins-Betreuung einer Gebärenden durch die Hebamme gehört in vielen Kliniken der Vergangenheit an. Stattdessen werden z.B. zentrale CTG-Monitoranlagen instal- liert. Eine Hebamme betreut oft mehr als eine Gebärende. Der Schmerzmittelverbrauch bzw. die PDA-Raten steigen mangels kontinuierlicher, individueller „Rund-um-Betreuung“. Intrapartale Immobilisation der Gebärenden führt häufig zu protrahierten Geburtsverläufen wegen Einstellungsanomalien, die dann aus Mangel an Zeit, Geduld und Erfahrung in einer Schnittentbindung münden. Um nicht missverstanden zu werden: ich schätze die Arbeit der Hebammen in den Kreißsälen und ihre Bemühungen um eine optimale Betreuung sehr! Aber auch ihrem Engagement sind reale - ökonomische - Grenzen gesetzt.

\section{Verlust von Wissen, Können und Erfahrung}

Die Einhaltung des Arbeitszeitgesetzes ist eine begrüßenswerte ärztliche Errungenschaft. Die Ausbildung scheint aber dadurch an Grenzen zu stoßen. Darüber hinaus dünnt sich der ärztliche „Mittelbau“ immer mehr aus. In vielen Frauenkliniken fehlen erfahrene, geburtshilflich versierte Fachärzte. Viele verlassen die Kliniken und lassen sich nieder. Ressourcen für die Ausbildung junger Ärztinnen und Ärzte wird so zum „Luxusgut“. Der Verlust an manuellen geburtshilflichen Techniken und an praktischer Erfahrung ist aus meiner Sicht ein wesentlicher Grund für die Steigerung der Sectioraten. Z.B. geht in Deutschland die Rate vaginal-operativer Entbindungen zurück in dem Maße, wie die Sectiorate steigt. Insbesondere die ForcepsEntbindung ist fast aus dem Repertoire verschwunden. Unter den hohen Sectioraten leidet das Erlernen manueller Techniken und das Sammeln von Erfahrungen, z. B. die manuelle Rotation des kindlichen Kopfes bei Einstellungsanomalien, vaginaloperative Entbindungsmethoden, die Manualhilfen bei Beckenendlagenentbindung, Leitung von vaginalen Mehrlingsgeburten oder von Geburten bei Zustand nach Sectio, die exakte intrapartale CTG-Beurteilung. Außerdem scheint sich das Wissen über die physiologischen Geburtsphasen immer mehr zu verwässern. Wie lang dauern durchschnittlich die latente und aktive Eröffnungsphase? Ab welcher Muttermundsweite geht die latente in die aktive Phase über? Welche stündliche Eröffnung des Muttermundes ist normal? Ab wann spricht man von einer protrahierten Austreibungsphase? Frage ich junge Kolleginnen und Kollegen, erhalte ich oft unbefriedigende Antworten.

\section{Gesellschaftliche Ursachen}

Gesellschaftliche Faktoren spielen eine nicht unwesentliche Rolle in dieser Entwicklung. Ein Grund liegt u.a. im steigenden Alter der Erstgebärenden. Damit verbunden sind die Zunahme der ernährungs- und lebensbedingten Zivilisationskrankheiten, z.B. Diabetes mellitus bzw. Gestationsdiabetes, präexistenter oder schwangerschaftsinduzierter Hypertonus, Adipositas, makrosome Neugeborene. Diese Krankheiten/Zustände können 
eine Schnittentbindung erforderlich machen. Späte Mutterschaft, hohes Anspruchsdenken an die Medizin und ein schier unbegrenzter Glaube an medizinische Wahlmöglichkeiten für jedermann fördert das egomanische Konsumverhalten. Der Kundengedanke der Marktwirtschaft hat längst in der Frauenheilkunde Einzug gehalten und fördert die Inanspruchnahme medizinisch nicht indizierter Leistungen. Das Spektrum reicht vom Kaiserschnitt aus „Angst“ bis hin zu plastisch-chirurgischen Operationen im Intimbereich aus Unzufriedenheit. Nichts wird dem Schicksal überlassen. Man glaubt, die Kontrolle über alle natürlichen Lebensvorgänge übernehmen zu können: von einer geplanten Empfängnis bzw. künstlich erzeugten Schwangerschaft bis hin zum geplanten Entbindungstermin. Die Missachtung von Naturgesetzen wird gesellschaftlich akzeptiert. Die negativen Auswirkungen auf die Gesundheit von Mutter und Kind werden dabei - z.T. in Unkenntnis - in Kauf genommen. Eine paranoide Fehlentwicklung, die wir verlassen müssen! Eine wichtige Feststellung im ACOG-Konsensus ist die Tatsache, dass die primäre Sectio am Termin reifen, gesunden Kindern hinsichtlich späterer Morbidität und Mortalität keinen Vorteil beschert. Im Bewusstsein der Bevölkerung besteht die Annahme, dass die Sectio der bessere, sichere Entbindungsweg für das Kind sei und allen Unbill abwende. Was für ein unphysiologischer Gedanke! Und diese Auffassung wird nicht selten von Frauen- und Kinderärzten vermittelt!

\section{Zukunftsvision}

Ich habe die Vision, dass sich die Rate der nicht medizinisch indizierten primären Sectiones reduzieren lässt. Die Geburtshelfer müssen sich wieder an wissenschaftlichen Daten orientieren und dieses aktuelle Wissen nicht nur in Fachjournalen, sondern auch in Medien für Laien verbreiten. Hier sind die jeweiligen Fachverbände gefordert. Ich kann mir vorstellen, dass die Deutsche Gesellschaft für Hebammenwissenschaft mit der Deutschen Gesellschaft für Gynäkologie und Geburtshilfe eine gemeinsame Presseerklärung herausgibt, in der auf die potenzielle Gesundheitsgefahr für Mutter und Kind durch eine nicht medizinisch indizierte (primäre) Sectio hingewiesen wird. Eindringlich müssen sie auf die derzeitige Fehlentwicklung hinweisen und Wege aufzeigen, um wieder zur „Normalität“ zurückzukehren. Für solche Aktionen können sicher auch Krankenkassen als Partner gefunden werden. Unerlässlich ist eine breite geburtshilfliche Ausbildung für Hebammen und für angehende Fachärzte. Hierfür müssen in den Ausbildungskliniken Ressourcen vorgesehen sein, die von den Kostenträgern finanziert werden. Die DRG's, die von Ausbildungskliniken abgerechnet werden, müssen einen „Ausbildungszuschlag“ enthalten. Ebenso muss die Eins-zu-Eins-Betreuung durch Hebammen wieder die Regel sein und über die DRG's finanziert werden. 


\section{Die Sicht einer Hebamme}

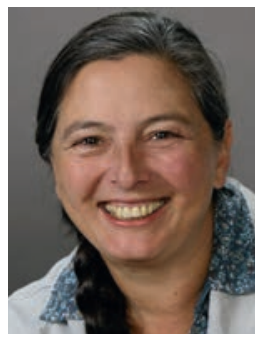

\section{Christiane Schwarz}

Hebamme, BSc Gesundheitswissenschaften, MSc Public Health (MPH)

Feldkamp 5, 31174 Schellerten

E-Mail: christiane-schwarz@gmx.de www.christiane-schwarz.de

Die neue Leitlinie hat für die Geburtshilfe in Deutschland - vielleicht sogar weltweit - das Potenzial, einen Paradigmenwechsel auszulösen. Ausgerechnet aus dem Land von „Save your love channel“, wo der einfache Kaiserschnitt in den letzten zehn Jahren zum „life event“ wurde und statt Hebammen „obstetric nurses" die Beinhalter für das Querbett richten, kommt nun eine Stellungnahme zur Förderung der physiologischen Geburt durch das Unterlassen von Interventionen.

Die beiden Fachgesellschaften American College of Obstetricians and Gynecologists (ACOG) und Society for Maternal-Fetal Medicine (SMFM) stellen fest:

\section{Bei hohen Sectioraten steigt die mütterliche Sterblichkeit} und den Kindern geht es nicht besser als bei deutlich niedrigeren Sectioraten.

Deshalb hinterfragen sie diese Geburtshilfe kritisch und bewerten die häufigsten Indikationen zur Sectio neu: „protrahierte Geburt/Geburtsstillstand“, „suspektes/pathologisches CTG“ und „Verdacht auf Missverhältnis/regelwidrige Einstellung“, sowie Beckenendlage und Mehrlinge.

Das Urteil lautet: (fast) alles Humbug.

\section{Im Einzelnen:}

1. Niemand weiß, wie lange normale Geburten dauern, wann sie beginnen, wie sie verlaufen und die sehr große Varianz beim Gebären ist komplett normal und ungefährlich. Also keine vorschnelle Diagnose und Behandlung von protrahierten Verläufen.

2. Das CTG ist ein unsicheres Instrument, deshalb darf die Diagnose „suspektes CTG“ keine Sectioindikation sein und deshalb müssen geburtshilfliche Teams gute Kenntnisse der (Patho-) Physiologie der fetalen Herztonmuster erwerben und permanent aktualisieren.

3. Regelwidrige Einstellungen sind durch manuelle Reposition des kindlichen Köpfchens oft therapierbar.

4. „Makrosomie“ ist eine Verdachtsdiagnose und als Sectioindikation unzulässig.

5. Zwillinge $>32$. SSW können sicher und gut vaginal zur Welt kommen, wenn der erste in Schädellage liegt.

6. Schwangeren mit BEL soll immer eine äußere Wendung angeboten werden.

Die Idee: Durch dieses lassen sich viele unnötige Kaiserschnitte vermeiden und die Anzahl von Re-Sectiones sinkt zwangsläufig mit.

\section{Was heißt das nun für uns?}

Wir haben in der Vergangenheit viele Empfehlungen aus den USA übernommen: in den 1970er und 1980er Jahren die routinemäßigen Zangengeburten mit Episiotomie im „Dämmerschlaf" und die programmierten Geburten, später die Sectio bei BEL und die Geburtseinleitung bei „Terminüberschreitung“, um nur einige zu nennen. Jetzt wendet sich das Blatt: wir dürfen, ja wir sollen Frauen beim Gebären wieder in Ruhe lassen.

Wie das in der Realität aussehen soll, wie Frauen beim Gebären am besten unterstützt werden können, wie wir die jahrzehntelange Verunsicherung und Angst von Schwangeren und GeburtshelferInnen wieder loswerden, wie wir wieder lernen, darin zu vertrauen, dass Geborenwerden und Gebären möglich ist - das herauszufinden kann ab jetzt unsere Aufgabe sein. Es ist die gemeinsame Aufgabe von Hebammen und Geburtshelferinnen, diese Wissenslücken kreativ und engagiert zu füllen und Konzepte zu entwickeln und umzusetzen.

Wer genügend Selbstbewusstsein hat, kann es gut aushalten, die Frauen in Ruhe zu lassen und sich mit ihnen über Geburten zu freuen, die aus ihrer Kraft und ohne medizinische Störung möglich waren. 